\title{
Towards understanding the ultra-fast dynamics of rhodopsin
}

\author{
Daniel P. Aalberts*, Fernando L. J. Vos, and Wim van Saarloos \\ Instituut-Lorentz for Theoretical Physics, Leiden University, P.O. Box 9506, 2300 \\ RA Leiden, The Netherlands
}

\begin{abstract}
The photoisomerization of rhodopsin in 200 femtoseconds is among the fastest and most efficient photochemical reactions known. We have developed a microscopic model to study rhodopsin's dynamics which retains the collective quantum mechanics of the $\pi$ electrons in the conjugated system. Our model is a generalization to three dimensions of Su, Schrieffer, and Heeger's model for polyacetylene $(\mathrm{CH})_{x}$. Model parameters are inferred from comparison with experiments and $a b$ initio calculations. The spatial structure and vibrational modes of the rhodopsin chromophore 11-cis retinal are calculated and shown to agree quite well with NMR and Raman spectroscopy measurements. Dynamics following photoexcitation are studied.
\end{abstract}

\section{INTRODUCTION}

Trying to understand how we see has occupied researchers for more than 100 years (ref 1). Vision in twilight conditions is attributed to rhodopsin, the complex formed by the chromophore 11-cisretinal bound in the pocket of a rod membrane protein called opsin. In the 1950s, vision was shown to arise from the rapid 11-cis to 11-trans isomerization of retinal (ref 2). The cones, responsible for color vision, also rely on the photoisomerization of 11-cis-retinal, differing only in the protein cages which surround the chromophore; however, in what follows, we will consider only rhodopsin's photochemistry.

We may be on the verge of understanding why rhodopsin is such an efficient and fast switch. What exactly do we mean by calling rhodopsin a switch? The optically active element, retinal, has two primary conformations-11-cis (rhodopsin or Rh) and 11-trans (bathorhodopsin or bRh)-and absorption of an optical photon $(\sim 5000 \AA)$ toggles the states. Recent experiments (ref 3$)$ show that, for rhodopsin, the isomerization is accomplished in a mere 200 femtoseconds, about the time it takes light to cross the width of a hair. Vibrational oscillations of the photoproduct cohere for a few ps (ref 4), much longer than the isomerization time. The photoisomerization is also very reliable, with $67 \%$ quantum efficiency; that, with a high stability against thermal fluctuations (400 years), yields the startling signal-to-noise ratio that makes the rod cells of our eyes essentially single photon detectors. The efficiency and speed are believed to be related quantities, in fact, since slower switches are observed to be less efficient (ref 5). Once a photon has flipped the switch, a sequence of dark reactions-driven thermally-follows, leading eventually to a nerve signal.

\section{PREVIOUS THEORY}

Birge and his colleagues (ref 6) did molecular dynamics calculations in the early 1980s and predicted isomerization times of $\sim 2.2$ ps. While this looks quite long in light of the current measurements with femtosecond lasers, the outcome of their calculation was already quite surprising as it was believed at that time to be too short for such a large conformational change. Their approach was a one spatial degree-of-freedom model; only rotations about the $\mathrm{C}_{11}=\mathrm{C}_{12}$ bond were considered, holding other relative coordinates fixed. In recent years, they have revised their calculation to include interaction with a counterion and now find it takes the excited state $400 \mathrm{fs}$ to reach the minima of the excited state surface from which it tunnels to $\mathrm{bRh}$ or $\mathrm{Rh}$, then $\sim 1 \mathrm{ps}$ to cool vibrationally to that local minimum. Experiments indicate an essentially barrierless process (ref 4).

Contemporaneously Su, Schrieffer, and Heeger (SSH) were investigating polyacetylene (PA), another material with double bonds in conjugation, and proposing their celebrated one-dimensional 

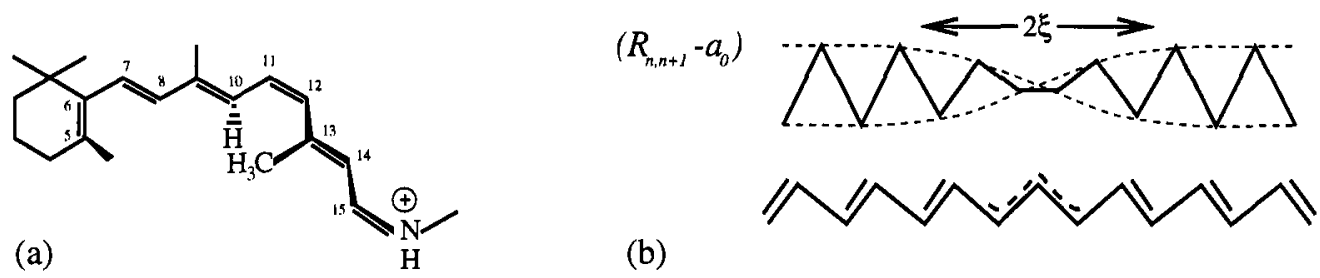

(b)

Fig. 1: (a) 11-cis-retinal. (b) A soliton of width $2 \xi$ : bond lengths $R_{n, n+1}$ oscillate about mean value $a_{0}$. Below, the same in standard chemical notation. Note that the soliton interchanges the bonding pattern (double-single $\leftrightarrow$ single-double).

model (ref 7, 8). SSH showed that solitons (Fig. 1b) appear in pairs, following photoexcitation, with astounding rapidity. A soliton is a compact and coherent (long-lived) lattice deformation pattern which smoothly bridges from a double-single to a single-double bonding pattern. Interestingly, solitonic lattice distortions draw energy levels into the gap ( $r e f 9,10,11)$. The conclusion is that the energy surface is sensitively dependent on the vibrating coordinates, not independent of time. Rh exits the Franck-Condon regime so quickly in part because forming solitons (in a few fs) reshapes the original energy landscape.

What we are attempting to do is to apply SSH's simple conceptual framework for PA to the more difficult problem of rhodopsin and its non-trivial three-dimensional structure. To fix the parameters of our model, we use measured vibration frequencies and we are aided by recent NMR experiments and $a b$ initio calculations which indicate the structure of retinal. One immediate advantage of our approach is that we may study (photo)excited electronic states; this is not possible in ab initio density functional approaches and is perhaps misleading in the approach of ref 6 because they neglect the effect of other spatial coordinates on the energy surface.

\section{MODEL}

In what follows, we will build upon the foundation laid by SSH in modeling the conjugated backbone with a tight-binding Hamiltonian in which the quantum mechanics for independent $\pi$ electrons is retained explicitly, while the other backbone forces arising from the $s p^{2}$ electrons and nuclear repulsion are treated with springlike effective potentials. Tight binding means that $\pi$ electrons may only hop to neighboring $p_{z}$ orbitals. Since electrons are much lighter than nuclei, we shall also assume that electrons instantaneously adjust to the wavefunctions that are evolving due to nuclear motion (the adiabatic or Born-Oppenheimer approximation). The essential electron-lattice coupling produces a dimerized lattice (alternating single and double bonds) and opens up a gap in the spectrum due to the Peierls instability. The gap, which determines much of what happens after photoexcitation, and the topological excitations seen in the SSH model, are believed to persist when electron-electron interactions are included (ref 9,12). We go beyond SSH in generalizing to a fully three-dimensional model, in explicitly considering finite chains, and in adding steric and electrostatic interactions specific to rhodopsin.

Our primary additional assumption in modeling three-dimensional conjugated polymers is of $s p^{2} p_{z}$ hybridization. That is to say that the $p_{z}$ orbital is perpendicular to the plane containing the three $s p^{2}$ orbitals. Using the Rh ground state coordinates determined in the ab initio calculations of Bifone, de Groot, and Buda (BGB, ref 13), we find near planarity of the $s p^{2}$ orbitals, even in the highly sterically strained regions. That gives us some confidence in this idealization.

Even with the simplifications, many elements contribute to our Hamiltonian. These can be explored one by one. The $\pi$-electron-lattice coupling is written as

$$
H_{e l}=-\sum_{s} \sum_{n=1}^{N-1}\left[\left(t-\alpha\left(R_{n, n+1}-a_{0}\right)\right) \cos \theta_{n, n+1}\right]\left[c_{n, s}^{\dagger} c_{n+1, s}+c_{n+1, s}^{\dagger} c_{n, s}\right],
$$

where $a_{0}=1.4 \AA$ is the average carbon-to-carbon spacing, $R_{i, j}=\left|\vec{R}_{i, j}\right|=\left|\vec{r}_{j}-\vec{r}_{i}\right|$ is the actual bond length between atoms $i$ and $j$, and the operator $\left(c_{i, s}^{\dagger} c_{j, s}\right)$ is the compact second-quantization notation for moving an electron with spin $s$ from site $j$ to site $i$ (with appropriate fermion properties implicit). Our intuition that electrons move more readily when the $p_{z}$ orbitals are close together is reflected in $H_{e l}$ for positive $\alpha$ and $t$. Twisting the $p_{z}$ orbitals out of alignment also reduces the likelihood of 
$\pi$-electron hopping (ref 14). We measure the degree of twisting with $\theta$, the angle between neighboring $p_{z}$ orbitals. The orientation of the $p_{z}$ orbital $\vec{\pi}$ is determined from the cross product

$$
\vec{\pi}_{n}=\vec{R}_{n-1, n} \times \vec{R}_{n, n+1}, \text { thus } \cos \theta_{n, n+1}=\frac{-\vec{\pi}_{n} \cdot \vec{\pi}_{n+1}}{\left|\vec{\pi}_{n}\right|\left|\vec{\pi}_{n+1}\right|} .
$$

The bond stretching, bond-angle bending, and kinetic terms are all included in the lattice part of the Hamiltonian:

$$
H_{l}=\frac{K_{\sigma}}{2} \sum_{n=1}^{N-1}\left(R_{n, n+1}-a_{0}\right)^{2}+\frac{K_{120}}{2} \sum_{n=2}^{N-1}\left(\Delta \phi_{n}\right)^{2}+\sum_{n=1}^{N} \frac{M_{n}}{2} \dot{\vec{r}}_{n}^{2}-\Gamma \sum_{n=1}^{N-1}\left(R_{n, n+1}-a_{0}\right),
$$

where $M_{n}$ is the mass of the $\left(\mathrm{C}_{n+4} \mathrm{H}\right)$ or $\left(\mathrm{C}_{n+4} \mathrm{Me}\right.$ ) group at position $n$ (in $\mathrm{Rh} n \leftrightarrow \mathrm{C}_{n+4}$ since conjugation begins at $\mathrm{C}_{5}$ ), where $\Gamma$ is a term which regulates the chain length for finite chains (ref 15), and where $\Delta \phi_{n}$ is the deviation in radians of the bond angle from $2 \pi / 3 \mathrm{rad}=120^{\circ}$. To first order

$$
\Delta \phi_{n} \approx \frac{R_{n-1, n+1}^{2}-R_{n-1, n}^{2}-R_{n, n+1}^{2}-R_{n-1, n} R_{n, n+1}}{\sqrt{3} R_{n-1, n} R_{n, n+1}} .
$$

So far, the model is generally applicable to conjugated polyene systems in three dimensions. To study rhodopsin, at least two additional terms must be included. One contribution stems from having a protonated Schiff base (PSB) at one end of the conjugated system. The nitrogen in the PSB is also $s p^{2} p_{z}$ hybridized (isoelectronic to carbon), but tends to attract electrons to itself more than a carbon. We may model this simply with an on-site potential

$$
H_{N}=V_{N} \rho_{N}, \text { where } \rho_{N}=\sum_{s} c_{N, s}^{\dagger} c_{N, s} .
$$

This contribution is simply $V_{N}$ times the electron density ( $-e \rho$ is charge density) at site $N$.

One further contribution to the Rh Hamiltonian is the steric interaction due to the crowding in the crook of the cis bond. Significant distortions result-taking the flat molecule and twisting it out of the plane (ref 13,16$)$. We model the steric interactions with an $r^{-12}$ potential

$$
H_{S t}=A\left(R_{\mathrm{H}, \mathrm{Me}}\right)^{-12} \text {, }
$$

where $R_{\mathrm{H}, \mathrm{Me}}$ is the distance from the hydrogen attached to $\mathrm{C}_{10}$ (at coordinate $\vec{r}_{\mathrm{H}}$ ) to the methyl group attached to $\mathrm{C}_{13}$ (at coordinate $\vec{r}_{\mathrm{Me}}$ ). The spatial coordinates of a side group are taken to be given by the backbone bonds such that angles between the group and the two backbone bonds are equal:

$$
\vec{r}_{\mathrm{Me}}=\vec{r}_{\mathrm{C}_{13}}+a_{C C}\left(\frac{\vec{R}_{\mathrm{C}_{13}, \mathrm{C}_{12}}}{R_{\mathrm{C}_{13}, \mathrm{C}_{12}}}+\frac{\vec{R}_{\mathrm{C}_{13}, \mathrm{C}_{14}}}{R_{\mathrm{C}_{13}, \mathrm{C}_{14}}}\right)\left|\frac{\vec{R}_{\mathrm{C}_{13}, \mathrm{C}_{12}}}{R_{\mathrm{C}_{13}, \mathrm{C}_{12}}}+\frac{\vec{R}_{\mathrm{C}_{13}, \mathrm{C}_{14}}}{R_{\mathrm{C}_{13}, \mathrm{C}_{14}}}\right|^{-1},
$$

where $a_{\mathrm{CC}}=1.54 \AA$ is the carbon-carbon distance. An analogous expression to (7) is used for the hydrogen, with $a_{\mathrm{CH}}=1.1 \AA$.

The complete Hamiltonian is a sum of these terms:

$$
H=H_{e l}+H_{l}+H_{N}+H_{S t} .
$$

The full dynamics of the atomic coordinates is performed by using the Feynman-Hellman theorem to calculate the forces, integrating forward, and recalculating at each step the electronic wavefunctions of the new configuration ( $r$ ef 15 ).

Our next challenge is to fix the parameters of the model. We take as our starting point the length over which the dimerization pattern varies in the ground state $\xi$, related to the dimensionless electron-phonon coupling parameter $\lambda$ by the asymptotic expression (ref 12)

$$
\xi / a_{0} \approx \frac{1}{4} \exp \left(\frac{1}{2 \lambda}+1\right), \text { with } \lambda=\frac{2 \alpha^{2}}{\pi K_{\sigma} t} .
$$



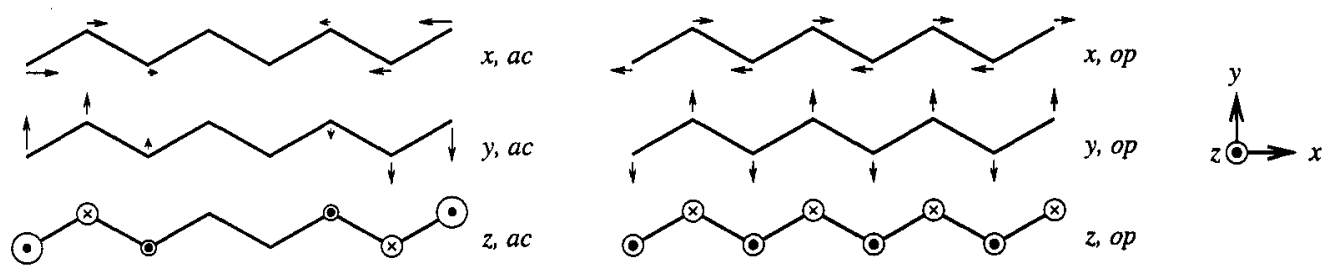

Fig. 2: Atomic displacement patterns for acoustical and optical modes. The frequency of each mode is given in (10).

Fitting to experiments done on PA, SSH found $\xi / a_{0}=7$. By (9), we find a relatively small electronlattice coupling $\lambda=0.21$.

\section{STRUCTURE AND VIBRATIONS}

We carried out a full analytical vibrational analysis of the trans-PA case of our model (chain in the $x y$-plane extending in the $x$ direction as shown in Fig. 2 and $H_{\mathrm{PA}}=H_{e l}+H_{l}$ ), for small electron-phonon coupling. The angular frequencies $\omega$ for the acoustical and optical modes are:

$$
\begin{aligned}
M \omega_{x, a c}^{2} & =\frac{32 K_{\sigma} \widetilde{K}_{120}}{K_{\sigma}+12 \widetilde{K}_{120}} Q_{x}^{2}, & M \omega_{x, o p}^{2} & =6 K_{\sigma} \lambda, \\
M \omega_{y, a c}^{2} & =0 Q_{y}^{2}+\frac{4}{3} \widetilde{K}_{120} Q_{y}^{4}, & M \omega_{y, o p}^{2} & =K_{\sigma}+12 \widetilde{K}_{120}, \\
M \omega_{z, a c}^{2} & =\frac{256 t}{3 \pi a_{0}{ }^{2}} Q_{z}^{2}, & M \omega_{z, o p}^{2} & =0
\end{aligned}
$$

where $\widetilde{K}_{120}=K_{120} a_{0}^{-2}$ and $Q_{x}=q_{x} \frac{\sqrt{3} a_{0}}{2}$ is a phase angle. Direct numerical simulation of these vibration modes confirms(10). The optical torsional and acoustic bending modes correspond merely to rotations out of the $x y$ plane and in the $x y$ plane, respectively; there is no restoring force for such displacements so they are zero-frequency modes. The calculation for $\omega_{x, o p}$ employs an asymptotic expansion in $\lambda$ for the energy and, as such, is only valid for small $\lambda$; the physical value of $\lambda=0.21$ is small enough that the asymptotic expansion is still good.

Since we are interested in determining the structure of $\mathrm{Rh}$ and its vibrational spectra, it is measurements of these quantities which we used to set the remainder of the parameters. Raman spectroscopy studies (ref 17) indicate optical modes of period $30 \mathrm{fs}\left(\approx 1100 \mathrm{~cm}^{-1}\right)$ for $x$ and period $25.8 \mathrm{fs}$ $\left(\approx 1290 \mathrm{~cm}^{-1}\right)$ for $y$. Optical frequencies differ little between PA or $\beta$-carotene. Using the formulae, we find $K_{\sigma}=47 \mathrm{eV} / \AA^{2}$ and $K_{120}=5.6 \mathrm{eV} /(\mathrm{rad})^{2} .(1 \mathrm{eV}=23 \mathrm{kcal} / \mathrm{mol}$.)

In Fig. 2, the acoustical modes are shown as uniform compression, bend, or twist. These deformations vibrate with period given by the slowest mode, the mode with wavelength twice the length of the molecule; thus, $Q_{\min }=\pi /(N-1)$. It is interesting to use these expressions to calculate the frequency of the slowest mode. The compression mode is fastest with a period of $110 \mathrm{fs}$. The torsional mode has period $220 / \sqrt{t / \mathrm{eV}}$ fs; for reasonable values of $t$ (discussed later) this is the next fastest. The slowest is the bending mode. Because $Q=\pi / 11$ yields primarily a rotation, we must look to the mode with $Q=2 \pi / 11$. We find this mode has period $385 \mathrm{fs}$, which compares favorably to the measured period of $550 \mathrm{fs}$ in the bRh photoproduct (ref 4). Therefore we attribute the vibrational ringing of the photoproduct to the bending, not the twisting mode. This leads to a new way of thinking about the photoisomerization: a quick nonlinear untwisting of the 11-cis bond excites many modes, but the observed coherent oscillations will be associated with the lowest frequency provided other frequencies are well separated from it. According to our calculations, this is mostly of bending $(y)$ character.

Different measurements lead to conflicting values of $t$. Based on the $\pi$ bandwidth of PA, SSH find $t=2.5 \mathrm{eV}$; however, with this value, steric strain is absorbed by bond-angle bending rather than by twisting-the ground state is flat, in contrast to the BGB structure. On the other end of the scale, values calculated by $a b$ initio studies of torsional spring constants around single and double bonds in hexatriene (ref 18) indicate $t \sim 0.4 \mathrm{eV}$. This would suggest an optical gap an order of magnitude too small. We take, at present, an intermediate value $t=1 \mathrm{eV}$ to achieve reasonably good agreement with the BGB structure, and a HOMO to LUMO gap of $0.62 \mathrm{eV}$. From (9) we find $\alpha=3.94 \mathrm{eV} / \AA$ and that fixes $\Gamma(\alpha, \lambda)=4.92 \mathrm{eV} / \AA(\operatorname{ref} 15)$.

The BGB ground state shows a solitonic dimerization pattern due to the electron withdrawal from the backbone by the PSB, see Fig. 3. Far from the nitrogen, bond lengths alternate uniformly. Near the 

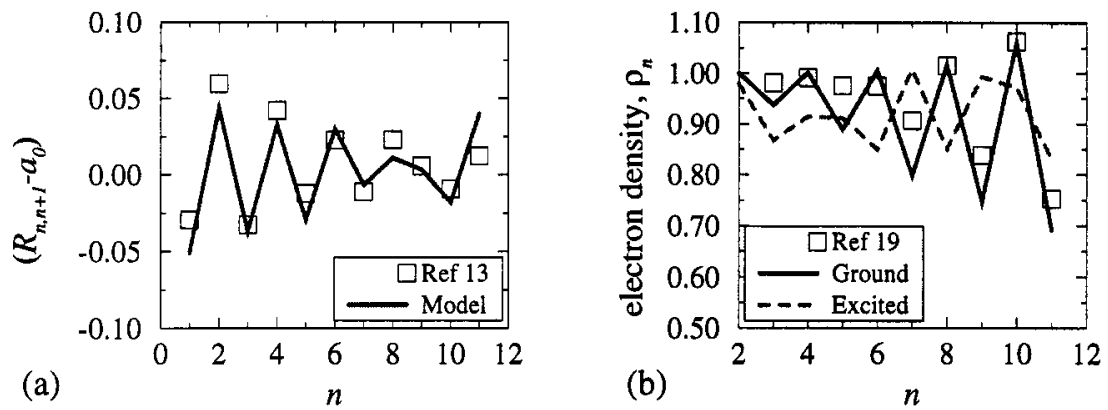

Fig. 3: (a) Dimerization pattern compared to $a b$ initio calculations (ref 13). Note the solitonic pattern (compare with Fig. 1b) centered near $n=9$. (b) Ground-state electron density compared with NMR measurement (ref 19). In the photoexcited state, the electron density near the nitrogen increases. Qualitative agreement is good.

nitrogen, this difference goes to zero-in fact, the formal $\mathrm{C}_{14}-\mathrm{C}_{15}$ bond really is short and the $\mathrm{C}_{15}=\mathrm{N}$ bond is long. The constant $V_{N}=-2.15 \mathrm{eV}$ produces a bond length alternation pattern consistent with that found by BGB (ref 13) and charge oscillations (ref 19). The constant $A=4000 \AA^{12} \cdot \mathrm{eV}$ was chosen to yield the measured spacing between the methyl and carbons 10 and 11:

$$
R_{\mathrm{Me}, \mathrm{C}_{10}}=\left\{\begin{array}{ll}
3.04 \AA & \text { model } \\
3.05 \pm 0.03 \AA & (\mathrm{ref} 16)
\end{array} \quad \text { and } R_{\mathrm{Me}, \mathrm{C}_{11}}=\left\{\begin{array}{ll}
3.10 \AA & \text { model } \\
3.05 \pm 0.05 \AA \quad(\text { ref 16) }
\end{array}\right),\right.
$$

and torsional angles consistent with $\mathrm{BGB}$ result:

$$
\left\{\theta_{\mathrm{C}_{10}, \mathrm{C}_{11}}, \theta_{\mathrm{C}_{11}, \mathrm{C}_{12},}, \theta_{\mathrm{C}_{12}, \mathrm{C}_{13}}\right\}= \begin{cases}\left\{15^{\circ}, 169^{\circ}, 8^{\circ}\right\} & \text { model } \\ \left\{11^{\circ}, 164^{\circ}, 14^{\circ}\right\} & (\text { ref 13) }\end{cases}
$$

Other torsional angles are near $0^{\circ}$ since no steric interactions are included to twist them. That $\theta_{\mathrm{C}_{11}, \mathrm{C}_{12}}$ is near $180^{\circ}$ indicates that it is a cis bond (2). Parameters $A$ and $V_{N}$ are not yet optimized for a best fit, but qualitative features are achieved.

This model, with these parameters, can be used to determine the three-dimensional ground-state structure and the dynamical properties of conjugated polymers (Rh, bRh, PA, cyclooctatetraene, etc). To get an impression of how reasonable these parameters are, let's compare the values we have derived with values used in chemical force-field calculations, deduced for hexatriene (ref 18). The hierarchy of interactions, most important for getting global structure right, is correct: bond length $\gg$ bond angle $\gg$ torsion. The compressional $K_{\sigma}$ and bond-angle $K_{120}$ spring constants we calculate agree well quantitatively with ref 18 . Our torsional $t$ constant is, as we indicated above, a couple of times larger than for hexatriene, but fits into the hierarchy.

The reader may wonder how our calculations differ from atomic force field approaches. The key is that we treat the collective response of the electrons which gives rise to the possibility for solitons, polarons, etc. We may also treat electronically excited states, which is also not possible in force-field calculations.

\section{PHOTOEXCITATION}

We have shown on finite $(N=50)$ chains that, following photoexcitation, the dimerization pattern interchanges in the center of the molecule persistently (ref 15). This means that in the center, until vibrational cooling or some other mechanism damps out the kinetic energy, what were double bonds become single bonds, and vice versa. In Fig. 4 we demonstrate that this is also the case for $\mathrm{Rh}$, in particular that the $\mathrm{C}_{11}=\mathrm{C}_{12}$ bond $(n=7)$ becomes a long bond. We imagine a mechanism in which the creation of soliton pairs (inverting the bond alternation pattern between) dynamically catalyzes the isomerization. The system starts sterically strained; by interchanging single and double bonds, the torsional stiffness at the $\mathrm{C}_{11}-\mathrm{C}_{12}$ bond is weakened. Without a large barrier, can the steric strain do the job of twisting the molecule to the less sterically strained bRh configuration? Our simulations, to date, do not realize this plausible scenario.

The charge density along the retinal backbone is another quantity which changes upon photoexcitation of Rh. In Birge's calculations (ref 6 ) the net charge, near the N, changes by about $0.25 e$. This effect is observed in our simulations (see Fig. 3). 


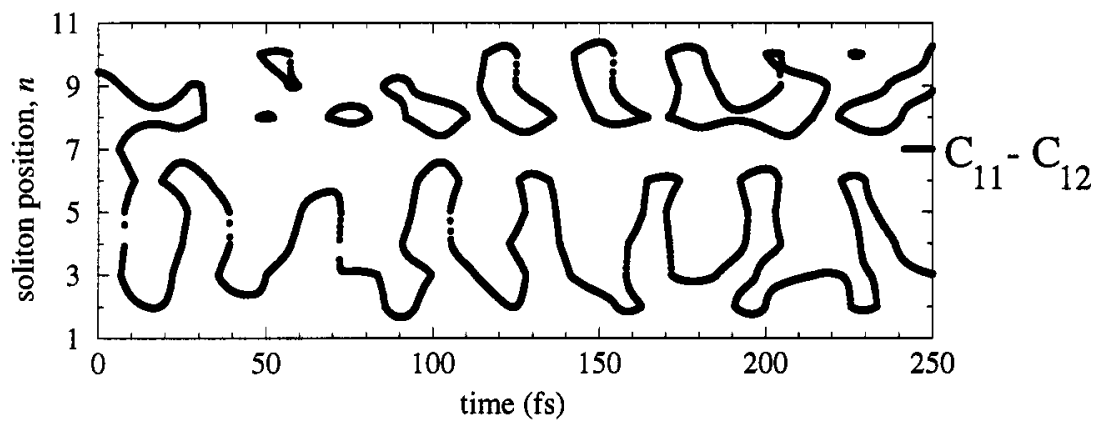

Fig. 4: After photoexcitation, the positions of solitons (where the dimerization pattern changes sign $R_{n, n+1}=$ $R_{\mathrm{C}_{n+4}, \mathrm{C}_{n+5}}=a_{0}$ ). Recall Fig. 1b. Note that $\mathrm{C}_{11}=\mathrm{C}_{12}$ bond becomes and remains a single bond after about $10 \mathrm{fs}$. Steric strain prevents returning to double-bond character in spite of the small conjugation length $(N=12)$.

Here we have ignored interactions of $\mathrm{Rh}$ with the protein. These interactions may assist in the photoisomerization and are a subject in our ongoing study. Yet within $200 \mathrm{fs}$, the protein cannot adjust much to the conformational change of the retinal, the exception being motion of the lysine residue bonded to the PSB. Protein interactions which may play an important role include: vibrational cooling, holding ring in place, deformation due to the lysine tugging on the end (perhaps related to the energy storage mechanism of $\mathrm{bRh}$ ), and the counterion. So long as experiments can fix the additional interaction parameters which need to be added, the modeling can proceed.

Although much work remains to be done, we are optimistic that a model of this type will provide the clues to understand rhodopsin's photoisomerization. Dynamical catalysis is one such novel mechanism inspired by this approach. Our prediction that bending is the dominant vibrational mode seen in the photoproduct is another outcome. The model is also of general utility in studying the three-dimensional structure and intermediates of other systems with $\pi$-conjugated backbones.

\section{ACKNOWLEDGMENTS}

We gratefully acknowledge discussions with Huub de Groot, Johan Lugtenburg, and Henk Eskes. We are also grateful to Angelo Bifone, Huub de Groot, and Francesco Buda for sharing their data before publication. D.P.A. and F.L.J.V. were supported by Stichting voor Fundamenteel Onderzoek der Materie (FOM), which is financially supported by the Nederlandse Organisatie voor Wetenschappelijk Onderzoek (NWO).

\section{REFERENCES}

* Present address: Center for Studies in Physics and Biology, Rockefeller University, New York, NY 10021-6399.

1. Reviewed briefly by: J. Nathans. Biochemistry 31, 4923 (1992).

2. R. Hubbard and A. Kropf. Proc. Nat. Acad. Sci. U.S.A. 44, 130 (1958).

3. R. W. Schoenlein, L. A. Peteanu, R. A. Mathies, and C. V. Shank. Science 254, 412 (1991).

4. Q. Wang, R. W. Schoenlein, L. A. Peteanu, R. A. Mathies, and C. V. Shank. Science 266, 422 (1994).

5. R. W. Schoenlein, L. A. Peteanu, Q. Wang, R. A. Mathies, and C. V. Shank. J. Phys. Chem. 97, 12087 (1993).

6. R.R. Birge and L.M. Hubbard. J. Am. Chem. Soc. 102, 2195 (1980). R.R. Birge and L.M. Hubbard. Biophys. J. 34, 517 (1981). R.R. Birge, C.M. Einterz, H.M. Knapp, and L.P. Murray. Biophys. J. 53, 367 (1988). R.R. Birge. Annu. Rev. Phys. Chem. 41, 683 (1990).

7. W.P. Su, J.R. Schrieffer, and A.J. Heeger. Phys. Rev. Lett. 42, 1698 (1979).

8. W.P. Su, J.R. Schrieffer, and A.J. Heeger. Phys. Rev. B 22, 2099 (1980).

9. A.J. Heeger, S. Kivelson, J.R. Schrieffer and W.P. Su. Rev. Mod. Phys. 60, 781 (1988).

10. $\mathrm{Yu} \mathrm{Lu}$, Solitons \& Polarons in Conducting Polymers (World Scientific, Singapore 1988).

11. D. Baeriswyl, in Theoretical Aspects of Band Structures and Electronic Properties of Pseudo-One-Dimensional Solids, ed. H. Kamimura, (Reidel 1985).

12. D. Baeriswyl, D.K.Campbell, and S. Mazumdar. In Conjugated Conducting Polymers, ed. H.G. Kiess (Springer Sol. St. Sci. 102, 1992).

13. A. Bifone, H.J.M. de Groot, and F. Buda. Unpublished.

14. W.A. Harrison. Electronic Structure and the Properties of Solids (W.H. Freeman, San Francisco, 1980).

15. F.L.J. Vos, D.P. Aalberts, and W. van Saarloos, Phys. Rev. B, 5314922 (1996).

16. P.J.E. Verdegem, P.H.M. Bovee-Geurts, W.J. de Grip, J. Lugtenburg, and H.J.M. de Groot. In preparation.

17. F. Inagaki, M. Tasumi, and T. Miyazawa. J. Raman Spect. 3, 335 (1973).

18. F. Negri, G. Orlandi, A.M. Brouwer, F.W. Langkilde, and R. Wilbrandt. J. Chem. Phys. 90, 5944 (1989).

19. H.J.M. de Groot. Personal communication. 